\title{
Evaluation of Covariates Influence on Urapidil Pharmacokinetics using Non-Linear Mixed Effect Model
}

\author{
M. Sundara Moorthi Nainar ${ }^{1,2}$, K. Ravisekhar ${ }^{1}$, D.Prabakaran ${ }^{3}$, V. Saji ${ }^{1}$, \\ T. Vijay ${ }^{1}$, S. Ashish ${ }^{1}$, V.Praveen Kumar ${ }^{1}$ and R.Sikandar Ali Khan ${ }^{1}$ \\ ${ }^{I}$ (Lupin Bioresearch Center, Pune 411021, Maharashtra, India) \\ ${ }^{2}$ (PhD Research Scholar, Department of Pharmaceutical Sciences, Jawaharlal Nehru Technological University, \\ Hyderabad - 500085, Andhra Pradesh, India) \\ ${ }^{3}$ (Ethics Biolabs, Chennai, Tamilnadu, India)
}

\begin{abstract}
Drug development is a very laborious, expensive and time consuming process. Inadequate pharmacokinetic knowledge on the drug candidate is one of the reasons for failure during drug development. The in-vivo absorbability of drugs categorized as BCS Class II is very difficult to predict because of the large variability in the absorption or dissolution kinetics. Urapidil comes under the category of BCS Class II. Thus, present study was aimed to assess the influence of covariates on pharmacokinetics of Urapidil from typical pharmacokinetics studies using population pharmacokinetic model. In this study one compartment model incorporating subject specific parameters was developed and evaluated. Results demonstrated that the one compartment absorption model without lag time under first order estimation method best describes the pharmacokinetics of Urapidil. The final model described the body weight influence on apparent oral clearance of Urapidil and $27.60 \%$ of the inter-individual variability was explained by the covariate body weight. Thus, it can be concluded that body weight was found to be the most important covariate for clearance of Urapidil. The projected model shall further developed in patients treated for Urapidil and results from this study should interpret cautiously while any dose adjustment for Urapidil treatment in patient population.
\end{abstract}

Keywords: Covariates, Eupressyl, NLME, Urapidil, Variability

\section{Introduction}

Drug development is a very laborious and expensive process. One of the major reasons for failure during the clinical phases of drug development is inadequate pharmacokinetic data on the drug candidate [1]. Pharmacokinetic variability is most commonly responsible for adverse drug reactions and therapy failure due to low drug exposures [2,3]. A number of factors can contribute to high variability in pharmacokinetic parameters [4]. The formulation factors that may impact on bioavailability and bioequivalence can be classified into two categories: (a) the first group belongs to factors that can affect drug dissolution or release which is considered as a prerequisite to the drug absorption process. (b) The second category comprises factors related to excipients or inactive ingredients which can influence drug stability, absorption and metabolism [5]. Population pharmacokinetics can be used to define the variability in plasma drug concentrations between individuals when standard dosage regimens are administered [6] and the identification and quantification of covariates, particularly using population pharmacokinetics is now seen as an integral part of drug development. However, many pharmaceutical companies go through unnecessary cycles of clinical studies involving formulation optimization without attention to the feasibility of reducing inter-individual variability and the source of such variation [7].

Many studies performed during drug development are aimed at identifying and quantifying between subject variability exhibit in drug exposure and response to improve the safety and efficacy of a drug agent. Variability is usually characterized in terms of fixed and random effects. The fixed effects are the population average values of pharmacokinetic parameters. The random effects quantify the amount of pharmacokinetic variability that is not explained by the fixed effects [8].

Knowledge of the variability of the biological systems is necessary to develop useful models. Thus, it is of crucial importance to identify the variables that contribute significantly in the process of drug absorption which will allow reliable predictions of drug absorption. Therefore integrated pharmacokinetic models take into account variables pertinent to pharmaceutical and physiological issues [5,9]. The advantages of population modeling include: a direct estimate of the population characteristics of pharmacokinetic parameters is obtained; studies can be modeled and optimized based on current knowledge; a smaller number of samples per individual, which is ethically desirable, can be used; combined analyses can be performed at the level of the raw data; complex models can be evaluated when data from many subjects are analyzed together [10].

The in-vivo absorbability of drugs categorized as BCS Class II is very difficult to predict because of the large variability in the absorption or dissolution kinetics [11]. Urapidil comes under the category of BCS 
Class II. The pharmacokinetic parameters are well defined after oral or intra-venous administration. Pharmacokinetic parameters after oral and intra-venous administration are similar and are linearly proportional to dose. In summary, the plasma concentration decreases for 10 minutes and then remains at that level for about 1 hour. The mean serum half-life of elimination is 2.7 hours. The plasma protein binding is $80 \%$. Two factors modify the pharmacokinetics of Urapidil: very old age, severe impairment of liver function [12].

To date, as per our understanding there is no research work published for Urapidil related to covariate modeling. Thus, present study was aimed to assess the influence of covariates on pharmacokinetics of Urapidil from typical pharmacokinetics studies using population pharmacokinetic model.

\section{Materials And Methods}

All materials used in this study purpose were complied with current United States Pharmacopeia (USP) and European Pharmacopoeia compendial specifications.

\subsection{Formulation}

Eupressyl 60mg (Urapidil Retard Capsules 60mg), Manufactured by Altana Pharma, France.

\subsection{Pharmacokinetic studies}

An open-label, randomized, fasting, single-dose, parallel/two way crossover studies were performed with 30 healthy, non-smoking, male subjects. The study protocols for Urapidil were approved by the Institutional Review Board at the clinical site. Written informed consent was obtained from all subjects prior to enrolment in the study. In each study period, after an overnight fast of at least $10 \mathrm{hrs}$, single oral dose of Urapidil Retard Capsules $60 \mathrm{mg}$ was orally administered with $240 \mathrm{~mL}$ of drinking water in sitting posture at ambient temperature in the morning, as per the randomization schedule. In each period, 21 blood samples were collected after dosing. Subjects were seated upright for the first two hours following drug administration and prohibited from any strenuous activity during housing period of the study. Plasma samples were stored at $-80^{\circ} \mathrm{C}$ before analysis. Plasma samples were separated and analyzed for Urapidil plasma concentrations.

\subsection{Analytical methods}

The in-house validated UPLC-MS/MS method was applied to determine the concentration of Urapidil in human plasma using Urapidil D4 as internal standard. The method was validated for selectivity, linearity, reproducibility, recovery, precision accuracy and stability. The measured concentrations for each subject for all the time points are calculated against the calibration curve prepared with known standards.

\subsection{Pharmacokinetic data analysis}

The Urapidil plasma concentration versus time data were evaluated using the Phoenix WinNonlin software version 6.3. Pharmacokinetic parameters Cmax, the maximum observed concentration, AUC0- $\infty$, Area under the plasma concentration versus time curve from time 0 to infinity, AUC the area under the concentration time curve, $\mathrm{CL} / \mathrm{F}$, total body clearance, $\mathrm{Vd} / \mathrm{F}$, volume of distribution and $\mathrm{t} 1 / 2$, half-life of the drug were determined for each subject and formulation.

\subsection{Development of base model}

The influence of covariates on the pharmacokinetics of Urapidil was evaluated analyzing the data pooled from three different pharmacokinetic studies. The final data for covariate modeling includes 30 south Asians, adult male healthy subjects. Covariate analysis was carried out following a two-stage approach.

In the first step, basic population pharmacokinetic model (without covariates) for Urapidil was performed applying nonlinear mixed effect model using Phoenix NLME version 1.2. Base model was used an experimental approach and focused on analyzing various structural models. An evaluation was undertaken to determine how well various types of pharmacokinetic models could describe the absorption profiles and variability in the existing data set. A first order absorption/zero order absorption or one compartment/two compartment models tested to explain variability associated with Urapidil pharmacokinetics. Inclusion of lag time and absorption rate constants $(\mathrm{Ka})$ were also assessed for improvement of estimations of the absorption processes. All compartment models were parameterized in terms of values of apparent oral clearance (CL/F) and apparent volume of distribution (V/F). Various models were evaluated and selected based on goodness of fit. Akaike Information Criterion (AIC) was compared using to differentiate models between non-hierarchical models in the selection of a structural model [13]. The basic structure of developed pharmacokinetic model is represented in Fig. 1. 


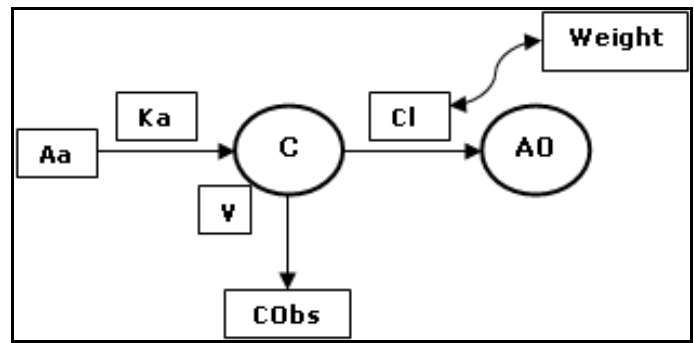

Fig. 1: Schematic representation of Urapidil pharmacokinetic model

$\mathrm{Aa}$ - Dose point (Urapidil 60mg)

$\mathrm{Ka}-$ Rate of absorption

C - Central compartment

$\mathrm{V}-$ Volume of distribution $\mathrm{Cl}$ - Clearance

A0 - Elimination compartment

CObs - Observed plasma Urapidil plasma concentration

\subsection{Development of Covariate model}

Covariate model was developed using the final base model for Urapidil. The subject-specific covariates that were tested to evaluate influence on PK parameter estimates as follows; four demographic data (age, height, body weight and BMI), one renal function marker (serum creatinine), five hepatic function markers (albumin, total protein, total bilirubin, SGOT and SGPT), one physiological variable (hematocrit) for Urapidil [14].

The step wise covariates search option was selected and this run mode performs an automatic stepwise forward or backward addition or deletion of covariates effects by adding one at a time to determine if they make a sufficient threshold improvement based on the specified criterion options. There were three options for this mode: the criterion on what to base the stepwise approach, the threshold for improvement in the criterion in order to add a covariate effect and the threshold to remove a covariate effect. The stepwise covariate search method used was the forward addition, backwards elimination where the structural model was used as a baseline and the covariate model was made increasingly complex. After each model estimation, the covariates were evaluated to see which one has the greatest improvement in the goodness of-fit statistic selected (-2LL, AIC, or $\mathrm{BIC})$ greater than the user-specified threshold. That covariate was added to the regression model for the structural parameter and the model was estimated [15].

In forward addition step covariates producing a decrease of $>3.84$ points in the AIC value were used for the evaluation and covariate was retained in the model when a significant drop observed in AIC (>-3.84), otherwise the next covariate was incorporated based on the ranking order. To obtain the full covariate model forward addition procedure was maintained until all covariates had been tested. For the backward deletion, a significance level of $\mathrm{P}<0.001$ was applied. Each covariate was removed from the model following backward deletion process. Those covariates did not produce a significant increase of 10.83 points in the AIC was removed from the model. This backward deletion process was stopped when the removal of each remaining covariate resulted in a significant deterioration of the model (AIC $>+10.83$ ) fit [16].

\subsection{Evaluation of final model}

Bootstrapping (re-sampling) method and visual predictive check (graphically compares the observations with their predictive distribution according to the model), method was adapted to compare the observations with their posterior predictive distribution according to the model [17].

For the bootstrap analysis, from the original obtained data 1000 replicates of parameter estimates were generated. Bootstrap distributions were compared with the final model using medians and the 2.5th and 97.5th percentiles of the estimates. For the visual predictive check, 100 subjects were simulated from the final model estimates. Calculated 5, 50 and 95th percentile and mean data was compared with the simulated data.

\section{Results And Discussion}

A total of 30 healthy adult human male subjects were enrolled in 3 different studies. Samples from all 30 subjects were analyzed to determine the plasma concentrations of Urapidil. Pharmacokinetic and statistical analyses were performed on data obtained from 30 subjects, who completed the studies as per the protocol. The pharmacokinetic parameters $\mathrm{C}_{\max }, \mathrm{AUC}, \mathrm{AUC}_{\mathrm{inf}}, \mathrm{T}_{\max }, \mathrm{CL} / \mathrm{F}, \mathrm{Vd} / \mathrm{F}, \mathrm{K}_{\mathrm{el}}$ and $\mathrm{t}_{1 / 2}$ for Urapidil were calculated by non-compartmental method using Phoenix WinNonlin Software (Version 6.3). Subjects' baseline characteristics are presented in Table 3.1. 
Table 3.1: Subjects' baseline characteristics

\begin{tabular}{|c|c|c|}
\hline Parameters & Mean \pm SD & Range \\
\hline Age (years) & $30.03 \pm 4.99$ & $20.00-39.00$ \\
\hline BMI $\left(\mathrm{kg} / \mathrm{m}^{2}\right)$ & $21.36 \pm 1.97$ & $18.00-24.70$ \\
\hline Height (cm) & $167.00 \pm 5.57$ & $154.00-176.50$ \\
\hline Weight (kg) & $59.73 \pm 6.09$ & $50.20-72.60$ \\
\hline Hematocrit (\%) & $42.18 \pm 2.86$ & $36.90-49.30$ \\
\hline Serum Creatinine (mg/dL) & $0.98 \pm 0.18$ & $0.73-1.37$ \\
\hline Total Bilirubin (mg/dL) & $0.52 \pm 0.20$ & $0.18-0.99$ \\
\hline Total Protein (gm/dL) & $7.25 \pm 0.51$ & $6.40-8.30$ \\
\hline Albumin (gm/dL) & $4.42 \pm 0.22$ & $4.03-4.94$ \\
\hline SGOT (U/L) & $19.91 \pm 6.76$ & $5.00-35.00$ \\
\hline SGPT (U/L) & $15.75 \pm 6.11$ & $5.00-30.00$ \\
\hline
\end{tabular}

\subsection{Evaluation of covariates influence on Urapidil pharmacokinetics}

A two-stage pharmacokinetic analysis approach was used in this study to evaluate the covariates influence on pharmacokinetics of Urapidil. Results from the first stage non-compartment analyses showed that obtained coefficient of variation for pharmacokinetic parameters indicate that variable pharmacokinetics was expected for Urapidil. Mean pharmacokinetic parameters are presented in Table 3.2.

Table 3.2: Mean obtained pharmacokinetic parameters for Urapidil

\begin{tabular}{|c|c|c|c|c|c|}
\hline Parameters & Mean & SD & Min & Max & CV\% \\
\hline$K_{\text {el }}(h r-1)$ & 0.12 & 0.01 & 0.09 & 0.18 & 22.014 \\
\hline $\mathbf{T}_{\text {half }}(\mathbf{h r})$ & 5.95 & 0.26 & 3.95 & 7.95 & 21.945 \\
\hline $\mathbf{T}_{\max }(\mathbf{h r})$ & 3.94 & 0.22 & 2.00 & 4.50 & 20.745 \\
\hline$C_{\max }(n g / m L)$ & 785.93 & 93.77 & 302.84 & 1976.20 & 30.420 \\
\hline $\operatorname{AUC}_{\text {INF }}(n g * h / m L)$ & 6679.39 & 561.74 & 2377.34 & 13642.48 & 34.045 \\
\hline$V d / F(L)$ & 0.09 & 0.01 & 0.03 & 0.21 & 32.157 \\
\hline $\mathrm{Cl} / \mathrm{F}(\mathrm{mL} / \mathrm{min})$ & 0.010 & 0.001 & 0.004 & 0.025 & 35.009 \\
\hline
\end{tabular}

The mean plasma concentration versus time profiles of Urapidil evaluated for all subjects. The figure displays mean plasma concentration data with standard deviation (SD) at each sampling time points (Fig. 2). The analysis showed evidence of variability in drug absorption processes in subject profiles. Fig. 2, Urapidil profile displayed a high variation during initial peak around 0-6 hrs post dose.

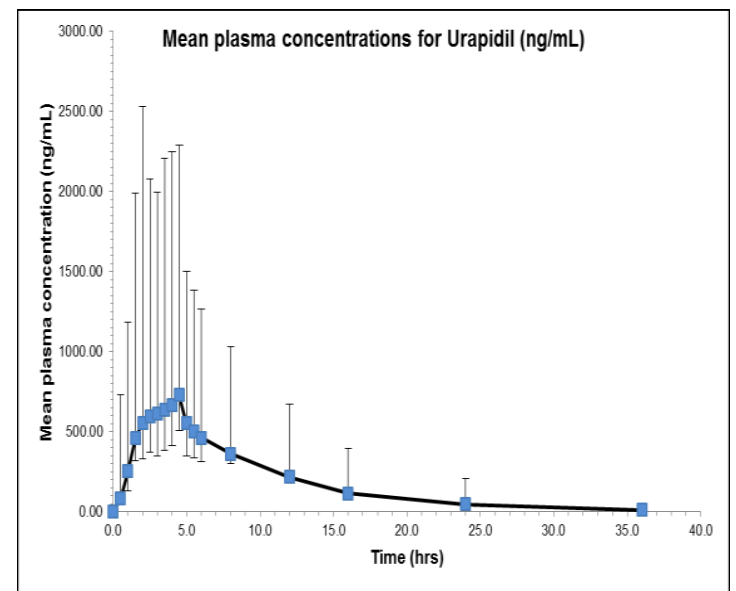

Fig. 2: Mean \pm SD Urapidil plasma concentrations vs. time profiles 


\subsection{Development of a population pharmacokinetic model}

A summary of base models with description considered in model development for Urapidil is outlined in Table 3.3. The best preliminary pharmacokinetic model was a one compartment model with first order absorption without lag-time which adequately describes the data, based on -2LL, AIC and BIC. Other absorption models were investigated including one or two compartment First-Order Conditional Estimation, Lindstrom-Bates, First-Order Conditional Estimation Extended Least Squares with and without lag time, which did not provide a significant improvement in the model fit (-2LL increased compared with the one compartment first order absorption models).

Table 3.3: Summary of base model used in development of a PK model for Urapidil

\begin{tabular}{|c|c|c|c|c|}
\hline Model & Model description & $-2 L L$ & AIC & BIC \\
\hline 3 & One compartment model without lag time FO & 7784.77 & 7804.77 & 7849.97 \\
\hline 5 & One compartment model with lag time FOCE_ELS & 8045.04 & 8061.04 & 8097.20 \\
\hline 6 & One compartment model with lag time FO_Tlag & 7932.98 & 7952.98 & 7998.17 \\
\hline 7 & Two compartment model without lag time FOCE_LB & 8208.58 & 8232.58 & 8286.81 \\
\hline 10 & Two compartment model with lag time FOCE_LB & 8057.32 & 8085.32 & 8148.59 \\
\hline 11 & Two compartment model with lag time FOCE_ELS & 8005.60 & 8033.60 & 8096.87 \\
\hline 12 & Two compartment model with lag time FO_Tlag & 7935.45 & 7963.45 & 8026.72 \\
\hline
\end{tabular}

FOCE L-B - First-Order Conditional Estimation, Lindstrom-Bates

FO - First Order

FOCE ELS - First-Order Conditional Estimation Extended Least Squares

-2LL - Twice the negative log likelihood

AIC - Akaike's Information Criterion

BIC - Bayes Information Criterion

\subsection{Covariates influence on outcome of Urapidil pharmacokinetics}

The one compartment absorption model without lag time under first order estimation method best describes the pharmacokinetics of Urapidil. Results showed that body weight was found to be the most important covariate for clearance of Urapidil. Since, other covariates did not reduce the AIC value significantly.

During initial model development height and weight was determined to have a significant influence when included in the one compartment model with first order absorption without lag time. Inclusion of height in the initial covariate analysis was influenced on apparent volume of distribution and weight on apparent clearance (CL/F) of Urapidil. However, while model optimization, inclusion of height and other covariates (age, height, body weight, BMI, serum creatinine, albumin, total protein, total bilirubin, SGOT, SGPT and hematocrit) did not produce a significant influence and failed to minimize successfully.

The final model described the body weight influence on apparent oral clearance (CL/F) of Urapidil and the weight range in the studied population was $50-72 \mathrm{~kg}$. The incorporation of body weight into the final model explained $9 \%$ of the inter-individual variability in $\mathrm{CL} / \mathrm{F}$ (decrease from 27 to $18 \%,(\Delta \mathrm{AIC}=+18$ when deleting the effect of body weight on clearance from the final model). This means that, relative to the observed interindividual variability, $27.60 \%$ of the inter-individual variability in this parameter was explained by the covariate body weight.

\subsection{Evaluation of final pharmacokinetic model}

This study may be one of the first attempts to develop a population pharmacokinetic model for Urapidil in healthy subjects. The proposed final population pharmacokinetic model incorporates the physiological aspects associated with Urapidil disposition. The projected model provides a basis for further development of a pharmacokinetic model in patients treated for Urapidil. The parameter estimation in the final model showed good precision and credible values and the AIC criterion was in favor of one-compartment model for Urapidil pharmacokinetics. The observed and predicted Urapidil plasma concentrations from the final model were distributed randomly across the line of unity when compared with base model (Refer Fig. 3). 


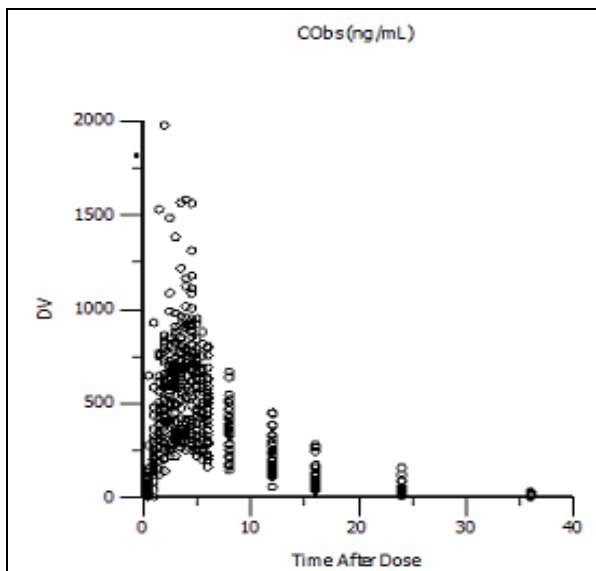

Obtained plasma Urapidil concentration vs Time

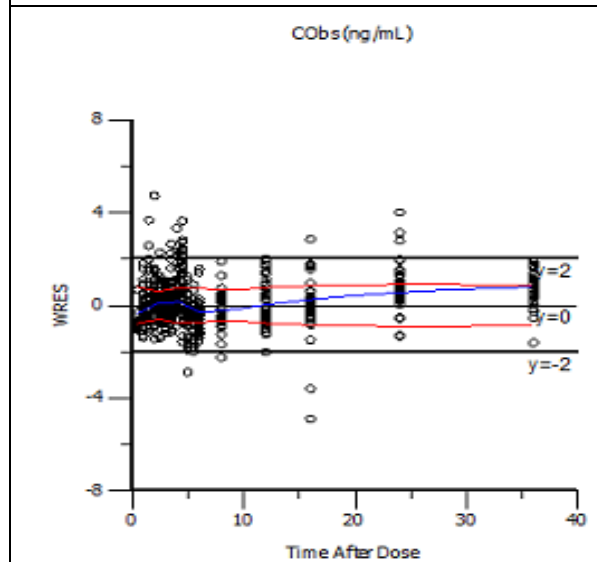

Weighted residuals for observed plasma Urapidil concentration versus Time

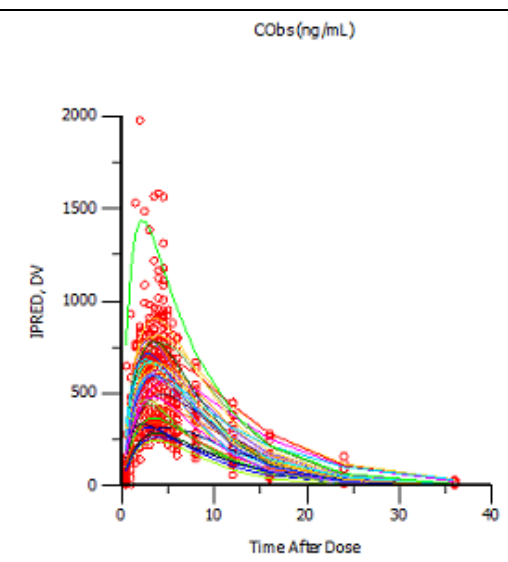

Predicted plasma Urapidil concentration vs Time

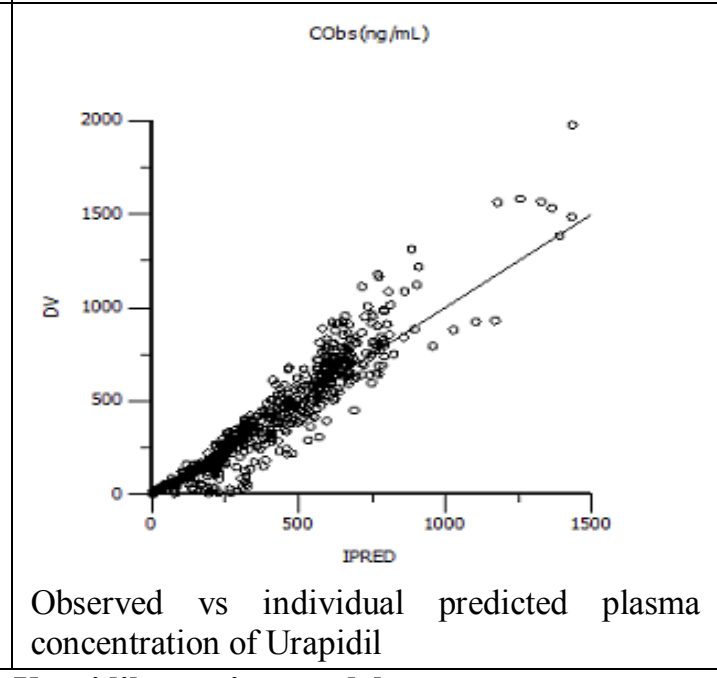

concentration of Urapidil

Fig. 3: Goodness of fit plots for Urapidil covariate model

The predictability of the final model for Urapidil was evaluated by the visual predictive check method and bootstrap re-sampling technique. In predictive check method calculated 5, 50 and $95^{\text {th }}$ percentile and mean data was comparable with the simulated data (Fig. 4). Thus, the results of covariate model demonstrating that the predictions of Urapidil plasma concentrations were on average unbiased. Overall, the final model was able to describe covariates influence on Urapidil pharmacokinetics. The estimates of the final model are outlined in Table 3.4.

Table 3.4: Results for Urapidil covariate model

\begin{tabular}{|c|c|c|c|c|}
\hline \multicolumn{5}{|c|}{ Final Model } \\
\hline Parameters & $\begin{array}{c}\text { Model } \\
\text { Estimate }\end{array}$ & $\begin{array}{c}\text { Residual } \\
\text { Variability }\end{array}$ & \multicolumn{2}{|c|}{ Inter-subject variability } \\
\hline $\mathrm{Ka}\left(\mathrm{h}^{-1}\right)$ & 1.1533 & 0.0456 & \multicolumn{2}{|c|}{13.9581} \\
\hline CL (Litres. $\left.h^{-1}\right)$ & 0.0641 & 0.0029 & \multicolumn{2}{|c|}{39.5732} \\
\hline V (Litres) & 0.0094 & 0.0004 & \multicolumn{2}{|c|}{22.2538} \\
\hline \multicolumn{5}{|c|}{ Bootstrap Estimates } \\
\hline Parameters & $\begin{array}{c}\text { Model } \\
\text { Estimate }\end{array}$ & $2.5 \%$ & Median & $97.5 \%$ \\
\hline $\mathbf{K a}\left(\mathbf{h}^{-1}\right)$ & 1.1521 & 1.0005 & 1.1515 & 1.3351 \\
\hline CL $\left(\right.$ Litres. $\left.^{-1}\right)$ & 0.0640 & 0.0564 & 0.0638 & 0.0710 \\
\hline V (Litres) & 4.8141 & 0.0049 & 0.3102 & 58.5819 \\
\hline dCldWeight & 1.1999 & 0.0019 & 1.2160 & 2.6450 \\
\hline
\end{tabular}

$\mathrm{Ka}$ - Rate of absorption

$\mathrm{Cl}$ - Clearance

$\mathrm{V}$ - Volume of distribution 


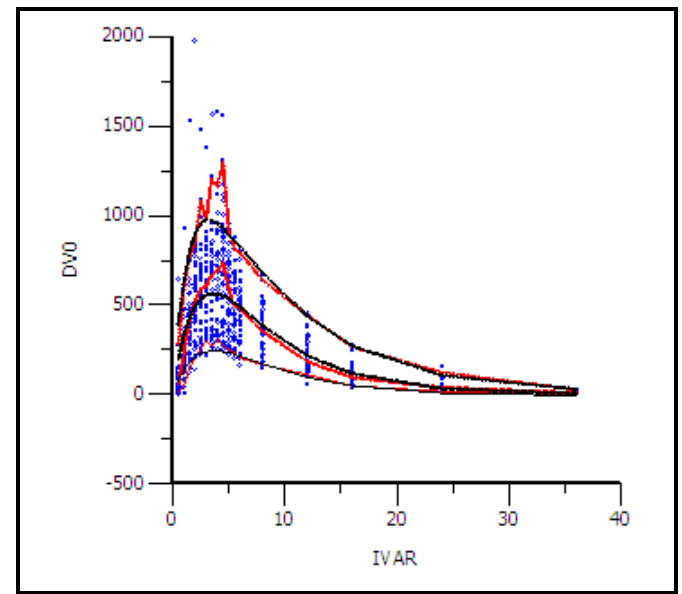

Fig. 4: Visual predictive check, Urapidil observed data compared with the $95^{\text {th }}, 50^{\text {th }}$ and $5^{\text {th }}$ percentiles for 100 simulated data sets

Blue dots represent observed Urapidil plasma concentration data. Solid red lines represent the percentiles (median, upper and lower percentiles i.e., 50\%, 05\% and 95\%) of the observed data. The solid black lines represent the median, upper and lower percentiles of the simulated data.

\section{Conclusion}

Till now for Urapidil several pharmacokinetic related articles have been described in the public domain. However, to the best of our knowledge covariate modeling in Urapidil pharmacokinetics is not yet reported in healthy volunteers. The final model described the body weight influence on apparent oral clearance of Urapidil. Thus, it can be concluded that body weight was found to be the most important covariate for clearance of Urapidil, since other covariates did not reduce the AIC value significantly. However, the projected model shall further developed in patients treated for Urapidil and results from this study should interpret cautiously while any dose adjustment for Urapidil treatment in patient population.

\section{Acknowledgements}

The authors thank Lupin Limited, India for technical assistance to complete this work. This paper is a part of thesis work for the grant of doctorate in Pharmaceutical Sciences from Jawaharlal Nehru Technological University, Hyderabad, India.

\section{References}

[1] Eva MD, Amo AT and Heikkinen JM. In vitro-in vivo correlation in p-glycoprotein mediated transport in intestinal absorption. Eur. J. Pharm. Sci. 36, 2009, 200-211.

[2] Ronald G. Hall II, Mark A. Swancutt, Claudia Meek, Richard D. Leff and Tawanda Gumbo. Antimicrob. Agents Chemother. 56, 2012,1502 .

[3] Jotam G. Pasipanodya, Shashikant Srivastava, and Tawanda Gumbo. Meta-Analysis of Clinical Studies Supports the Pharmacokinetic Variability Hypothesis for Acquired Drug Resistance and Failure of Antituberculosis. Therapy Failure and Pharmacokinetic Variability. CID. 55, 2012, 169-177

[4] Barbara M. Davit et. al., Highly Variable Drugs: Observations from Bioequivalence Data Submitted to the FDA for New Generic Drug Applications. The AAPS Journal.10, 2008, 148-156.

[5] Vangelis Karalis, Panos Macheras, Achiel Van Peer and Vinod P. Shah: Bioavailability and Bioequivalence:Focus on Physiological Factors and Variability. Pharm Res. 25, 2008, 1956-1962.

[6] L. AARONS, Population pharmacokinetics: theory and practice. Br. J. Clin. Pharmac. 32, 1991, 669- 670.

[7] Masoud Jamei, Gemma L Dickinson and Amin Rostami-Hodjegan: A Framework for Assessing Inter- individual Variability in Pharmacokinetics Using Virtual Human Populations and Integrating General Knowledge of Physical Chemistry, Biology, Anatomy, Physiology and Genetics: A Tale of 'Bottom- Up' vs 'Top-Down' Recognition of Covariates. Drug Metab. Pharmacokinet. 24, 2009, 53-75.

[8] DR Mould and RN Upton. Basic Concepts in Population Modeling, Simulation, and Model-Based Drug Development. CPT Pharmacometrics Syst. Pharmacol. 1, e6; doi:10.1038/psp.2012.4.

[9] Wade, J. R., A. W. Kelman, C. A. Howie, and B. Whiting, "Effect of Misspecification of the Absorption Process on Subsequent Parameter Estimation in Population Analysis," J Pharmacokinet Biopharm 1993; 21:209-222.

[10] Leon Aarons et. al., Role of modelling and simulation in Phase I drug development. Eur J Pharm Sci. 13, 2001, 115-122.

[11] B. Basanta Kumar Reddy and A. Karunakar, Biopharmaceutics Classification System: A Regulatory Approach. Dissolution Technologies. 2011, 31-37.

[12] Urapidil Nordic Pharma $25 \mathrm{mg} / 5 \mathrm{ml}$ and $50 \mathrm{mg} / 10 \mathrm{ml}$ Solution for injection, Public Assessment Report. FR/H/342/01-02/MR. Applicant: Nordic Pharma. Date of the PAR: December 2008.

[13] Catherine. Development of population PK model with enterohepatic circulation for mycophenolic acid in patients with childhoodonset systemic lupus erythematosus. Br J Clin Pharmacol. 7, 2011,727-740. 
[14] Isabelle K. Delattre, Flora T. Musuamba, Joakim Nyberg, Fabio S. Taccone, Pierre-Francxois Laterre, Roger K. Verbeeck, Fre' de'rique Jacobs and Pierre E. Wallemacq. Population Pharmacokinetic Modeling and Optimal Sampling Strategy for Bayesian Estimation of Amikacin Exposure in Critically Ill Septic Patients. Ther Drug Monit. 32, 2010,749-756.

[15] User's Guide, Phoenix ${ }^{\circledR}$ WinNonlin ${ }^{\circledR} 6.3$, Phoenix ${ }^{\circledR}$ Connect 1.3 and Phoenix ${ }^{\circledR}$ NLME 1.2, Pharsight Corporation 2005-2012, Certara, L.P.

[16] Thorsten Lehr et. al., Population pharmacokinetic modelling of NS2330 (tesofensine) and its major metabolite in patients with Alzheimer's disease. Br J Clin Pharmacol. 64, 2007, 36-48.

[17] Karl Brendel et. al., Are Population Pharmacokinetic and/or Pharmacodynamic Models Adequately Evaluated? A Survey of the Literature from 2002 to 2004. Clin Pharmacokinet. 46, 2007, 221-234. 